\title{
Inverse and Reciprocity Methods for Machinery Noise Source Characterization and Sound Path Quantification Part 1: Sources
}

\author{
Jan W. Verheij \\ TNO Institute of Applied Physics, P. O. BOx 155, 2600 AD DELFT, The Netherlands
}

In this article and in a forthcoming companion article some recently explored experimental approaches to the characterization of the noise source strength of machinery and to the ranking of transmission paths are reviewed. They form an addition to the more conventional approaches of the analysis of noise control problems in terms of source-transmission path-receiver schemes. In this first article source strength descriptors are defined both for airborne and for structure-borne sound. Their common basis is that the physical sources are modelled in terms of fictitious elementary sources, such as acoustical monopoles or mechanical point forces. In three of the four methods discussed, the strength of these equivalent substitution sources is determined indirectly. Advantages as well as limitations are considered. One practical advantage concerns the feature that the proposed descriptors are much less affected in strongly different installation environments than the more conventional source strength descriptors. Another practical advantage is that the use of elementary substitution sources as source models facilitates the very convenient application of experimental reciprocity techniques for transmission path ranking. Validation experiments related to applications in ships, road vehicles and office machines are briefly discussed.

\section{INTRODUCTION}

A conventional approach for the analysis of noise control problems uses the source-transmission path-receiver scheme. In many situations this type of analysis is complicated by the fact that there is a multitude of simultaneous sources. Moreover, even for a single noise source like a machine, there can be a multitude of partial sources and "parallel" transmission paths. Therefore, the development of cost-effective noise reduction strategies often requires detailed knowledge of the contributions of the partial sources and transmission paths to the radiated sound at distant receiver positions. Generally speaking, this type of analysis requires a system modelling in terms of inputs $I$, which genuinely characterize the sources themselves and of output-input ratios $O / I$. These latter are transfer functions $T F$, which characterize the transmission paths. Such an analytical approach facilitates the effective specification of required noise source quietening and of improved sound or vibration isolation in one or more paths.

The treatments in this article will be limited to systems with supposedly linear behaviour, i.e. to systems for which the output response may be modelled as a linear superposition of all contributions of the partial sources and transmission paths. Then in loose mathematical terms the following model equations apply:

- single source, single transmission path:

$$
O=T F \times I
$$

- single source, multiple transmission paths, e.g.:

$$
O=\sum_{j=1}^{m}\left(T F_{j} \times I\right)
$$

- multiple sources, multiple transmission paths, e. g.:

$$
O=\sum_{i=1}^{n}\left\{\sum_{j=1}^{m}\left(T F_{i j} \times I_{i}\right)\right\} .
$$

As is conventional practice for linear system analysis, frequency domain formulations will be used in this article. However, the above equations may then still represent a variety of models. They cover, for example, discrete frequency formulations, which take into account all relevant phase relationships. But they also cover relative bandwidth formulations (e.g. 1/3-octave bands), which use mean squared bandfiltered inputs and outputs and frequency band averages of the squared magnitude of frequency response functions $T F(f)$.

This first article is devoted to a discussion of some rather unconventional source descriptors $I$, for situations where more conventional approaches become rather impractical or are not compatible with practical methods for transfer function determination.

In a second article some applications of these source strength descriptors to the problem of path ranking will be discussed $^{1}$. In that article the experimental elegance will be underlined of reciprocity measurements for the determination of transfer functions $T F$, which are compatible with the newly defined source descriptors $I$. 